\title{
Condensation phenomena in nonlinear drift equations
}

\author{
José A. CARrillo, Marco Di Francesco And Giuseppe Toscani
}

\begin{abstract}
We study non-negative, measure-valued solutions to nonlinear drifttype equations modelling concentration phenomena related to Bose-Einstein particles. In one spatial dimension, we prove existence and uniqueness for measure solutions. Moreover, we prove that all solutions blow up in finite time leading to a concentration of mass only at the origin, and the concentrated mass absorbs increasingly the mass converging to the total mass as $t \rightarrow \infty$. Our analysis makes a substantial use of independent variable scalings and pseudo-inverse functions techniques.
\end{abstract}

Mathematics Subject Classification (2010): 35B40 (primary); 35B44; 35L65; $35 \mathrm{Q} 40$ (secondary).

\section{Introduction}

In this paper we perform a rigorous study of non-negative measure solutions of the nonlinear drift-type equation

$$
\partial_{\tau} f=\nabla_{v} \cdot\left(v f\left(1+f^{\gamma}\right)\right), \quad \gamma>0,
$$

posed on $v \in \mathbb{R}^{d}$, where $d \geq 1$, and $\tau \geq 0$, with initial condition $f_{I} \in L_{+}^{1}\left(\mathbb{R}^{d}\right)$.

In the case $\gamma=1$, the nonlinear drift on the right-hand side of equation (1.1) appears as the confinement operator in the Fokker-Planck equation introduced by Kaniadakis and Quarati $[18,19]$, who proposed a correction to the linear drift term of the Fokker-Planck equation to account for the presence of quantum indistinguishable particles, bosons or fermions. There, the evolution equation for bosons density

JAC acknowledges support from the Royal Society by a Wolfson Research Merit Award and by the EPSRC grant with references EP/K008404/1.

MDF is supported by the FP7-People Marie Curie CIG (Career Integration Grant) Diffusive Partial Differential Equations with Nonlocal Interaction in Biology and Social Sciences (DifNonLoc) and by the 'Ramon y Cajal' sub-programme (MICINNRYC) of the Spanish Ministry of Science and Innovation, Ref. RYC-2010-06412.

JAC and MDF were partially supported by the project MTM2011-27739-C04-02 DGI (Spain).

Received July 8, 2013; accepted in revised form February 11, 2014.

Published online February 2016. 
has been postulated in the form

$$
\partial_{\tau} f=\nabla \cdot[\nabla f+v f(1+\epsilon f)], \quad \epsilon>0 .
$$

The fundamental assumption leading to the correction in the drift term of the linear Fokker-Planck equation is established in [6] by imposing that a state of congestion emerges when the mean distance between neighboring particles is comparable to the size of the quantum wave fields in which the particles are embedded. In the case of a gas composed by Bose-Einstein identical particles, (according to quantum theory) the probability that a new particle will enter the velocity range $d v$ increases when the neighboring particles are of the same type. More precisely, the presence of $f(v) d v$ particles per unit volume increases this probability in the ratio $1+\epsilon f(v)$, for some $\epsilon>0$. tion [6]

By a direct calculuation, one can easily check that the Bose-Einstein distribu-

$$
f_{\lambda}(v)=\frac{1}{\epsilon}\left[e^{|v|^{2} / 2+\lambda}-1\right]^{-1}
$$

satisfies the equation

$$
\nabla f_{\lambda}(v)+v f_{\lambda}(v)\left(1+\epsilon f_{\lambda}(v)\right)=0
$$

for any fixed positive constant $\lambda$, and it is therefore a stationary state for the equation (1.2). The constant $\lambda$ is related to the mass of Bose-Einstein distribution

$$
m_{\lambda}=\int_{\mathbb{R}^{d}} \frac{1}{\epsilon}\left[e^{|v|^{2} / 2+\lambda}-1\right]^{-1} d v
$$

and, since $m_{\lambda}$ is decreasing as $\lambda$ increases, the maximum value of $m_{\lambda}$ is attained at $\lambda=0$. If $d=3$, the value

$$
m_{c}=m_{0}=\int_{\mathbb{R}^{3}} \frac{1}{\epsilon}\left[e^{|v|^{2} / 2}-1\right]^{-1} d v<+\infty
$$

defines a critical mass.

One of the main problems in kinetic equations relaxing towards a stationary state characterized by the existence of a critical mass is to detect a singular part (the condensate) for the solution when the initial distribution has a supercritical mass $m>m_{c}$. In general this phenomenon is heavily dependent of the dimension of the physical space. For instance, in dimension $d \leq 2$ the maximal mass $m_{0}$ of the Bose-Einstein distribution (1.3) is $+\infty$, and the eventual formation of a condensate is lost.

The kinetics of Bose-Einstein condensation - in particular, the way in which the Bose fluid undergoes a transition from a normal fluid to one with a condensate component - has been the object of various investigations [11-13, 16, 22,25-27]. The results in the aforementioned literature are mainly based on study of kinetic equations, like the quantum Boltzmann equation and the Boltzmann-Nordheim 
equation, which describes the dynamics of weakly interacting quantum fluids, see also the recent [14].

Several kinetic models for Bose-Einstein particles have been proposed and studied in the literature. In particular, a related model described by means of Fokker-Planck type non linear operators has been proposed by Kompaneets [20] to describe the evolution of the radiation distribution in a homogeneous plasma when radiation interacts with matter via Compton scattering. This equation has been exhaustively studied in [10]. It should be noticed that the Kompaneets equation also features a nonlinear drift of similar type as in (1.1).

The study of the mathematical theory for the Fokker-Planck equation (1.2), both in the bosons and fermions cases, is quite recent, and still partly open. Existence of solutions for the fermions case has been investigated in [3]. For bosons, equation (1.2) has been studied in dimension one of the velocity variable in [4]. In this case, however, the equilibrium Bose-Einstein density is a smooth function, which makes it possible to prove exponential convergence to equilibrium by relying on standard entropy methods. The three-dimensional problem, and the eventual formation of a condensed part in the solution of the equation (1.2), has been recently investigated in [28], where it is shown that, for a given initial supercritical mass, there is blow-up in finite time of the solution. However, nothing has been proven on the behavior of the solution after the blow-up time. It is clear that both the finitetime blow up proven in [28], and the possible subsequent formation of a condensed part, are due to the presence of the nonlinearity in the drift equation. Indeed, the dissipation of energy in the linear drift equation is not enough to produce blow up in finite time, and concentration of mass is obtained only as time goes to infinity.

While the case $\gamma=1$ is the most relevant one from the physical point of view (due to its relationship with the Bose-Einstein distribution), Fokker-Planck equations of type (1.1), with $\gamma>1$ and with linear diffusion have been considered in [2], with the aim of finding minimizers to sub-linear entropies. There, it has been shown that a suitable coupling of the degree of nonlinearity of the drift and of the space dimension ( $\gamma>2$ if $d=1$ ) allows the equilibrium profile to have a singular part, which is localized in space in a precise way.

The analysis of $[2,28]$ motivates the study of equation (1.1), both in dimension one, and in higher dimensions of the velocity variables. By enlightening its main properties - in particular towards the direction of the formation of condensation in finite time, and the continuation of measure solutions for all times - we hope to shed a light on the main problem of the formation of a condensed part in the case of the complete Fokker-Planck equation with linear diffusion (1.2).

The first step in our analysis is to reduce (1.1) to the new equation

$$
\partial_{t} \rho-\operatorname{div}\left(x \rho^{1+\gamma}\right)=0,
$$

via a time dependent scaling which removes the linear drift part, see Section 2 below. The main advantage of (1.4) is that the nonlinear drift term becomes homogeneous.

In one space dimension, this very simple key observation paves the way to our next key remark, namely that the Cauchy problem for the equation (1.4) can be 
related to the Cauchy-Dirichlet problems for a classical scalar conservation law

$$
\left\{\begin{array}{lll}
u_{t}+\left(\frac{1}{1+\gamma} u^{1+\gamma}\right)_{\xi}=0 & \text { if } & \xi<0 \\
u_{t}-\left(\frac{1}{1+\gamma} u^{1+\gamma}\right)_{\xi}=0 & \text { if } & \xi>0
\end{array}\right.
$$

with boundary datum $u(0, t)=0$, via the scaling

$$
\begin{aligned}
u(\xi, t) & =x^{\prime}(\xi) \rho(x(\xi), t), & & \xi \in \mathbb{R}, \quad t \geq 0 \\
x(\xi) & =\operatorname{sign}(\xi) \frac{1}{1+\gamma}(\gamma|\xi|)^{(1+\gamma) / \gamma}, & & x^{\prime}(\xi)=(\gamma|\xi|)^{1 / \gamma} .
\end{aligned}
$$

see Section 3 below.

The scaling (1) can be easily justified in case of a bounded solution $\rho \in L^{\infty}$ of (1.4), which corresponds to $u(0, t)=0$. Then, when the left and right traces of $u$ at $\xi=0$ become positive, $u$ and $\rho$ start losing mass. Based on the previous results in the literature, and having not included diffusion in the model, we clearly expect that the lost mass gets concentrated. Hence, the scaling (1) becomes the main tool to explore condensation phenomena for (1.1) via the auxiliary equation (1.4).

On the other hand, this requires a global-in-time well-posedness theory for measure solutions for (1.1), in a way to embrace also entropy solutions a la OleinikKružkov [21,24], at least for short times. The achievement of such a goal constitutes the main result of this paper, and it is rigorously stated in Definition 5.1 and in Theorem 5.2 in Section 5, the main difficulty being represented by the nonlinearity $\rho^{1+\gamma}$ in the drift term, which renders the definition of a Dirac delta type solution a non trivial task. The main tool used to overcome this difficulty is to consider the pseudo-inverse formulation of the equation (1.4), see Section 4, which allows to consider constant solutions in the pseudo-inverse variable corresponding to concentrated parts in the solution to (1.4).

Based on our existence and uniqueness result in Theorem 5.2, we then analyse the large-time behaviour of the measure solutions. More precisely, under a suitable restriction on the initial conditions (still in a context of large data) we prove that all measure solutions to (1.4) concentrate in a finite time, and the concentrated mass is strictly increasing after the blow-up time. Finally, we prove that all the total mass concentrates as $t \rightarrow+\infty$. This is proven in Theorem 6.1. A quite enlightening special solution is described in the Example 3.6, in which we see a bounded initial data evolving towards a condensate state as follows: at some time $t^{*}$ the profile of the solution becomes unbounded at zero, but still integrable. Immediately after $t^{*}$, the solution concentrates gradually, and all the mass is concentrated in infinite time.

It is interesting to notice that (partially) explicit solutions of the pseudo-inverse equation (4.2) are provided in Section 4 by the method of characteristics for fully nonlinear PDEs, in the spirit of Hamilton-Jacobi type equations, see [23]. A possible way to achieve existence and uniqueness for that problem was then the use of the theory of viscosity solutions, see e.g. [7]. On the other hand, the special structure of the equation and its relation to the scalar conservation law (1.5) allow to 
prove existence and uniqueness in a more direct way, and this gives as a by product the equivalence with Oleinik's entropy solutions of (1.4) for short times.

The use of the pseudo-inverse variable for transport equations is not new, see the seminal papers [5, 17] and [29] for further references. The main advantage of such approach is to give sense to measure solutions in an easy way, as concentrated measures become constant states in the pseudo-inverse variable. The use of such approach to 'nonlinear conservation law' like models such as (1.1) is, to our knowledge, totally new. In particular, the main novelty in our approach is the combination of the classical concept of entropy solution for a scalar conservation law with the concept of measure (concentrated) solution to a transport PDE.

The paper is organized as follows. In Section 2 we present the scaling needed to write (1.4), and provide a short time existence result of smooth solutions via characteristics, which holds in arbitrary space dimension. In Section 3 we analyse the one-dimensional case, and explain in detail the relation with the classical conservation law model (1.5). Here we provide also the special solution mentioned above. In Section 4 we introduce the use of the pseudo-inverse variable, and provide explicit solutions via the method of characteristics for fully nonlinear PDEs. In Section 5 we provide out main results in Theorem 5.2, which is stated and proven in terms of the solution $\rho$ to (1.4), see also Corollary 5.3 for a statement in terms of the solution $f$ to $(1.1)$. In Section 6 we prove the results about the qualitative and asymptotic behaviour mentioned before.

\section{The multi-dimensional case}

Throughout the whole paper, $\mathcal{P}\left(\mathbb{R}^{d}\right)$ denotes the space of probability measures on $\mathbb{R}^{d}$, and $\mathcal{L}^{d}$ denotes the Lebesgue measure on $\mathbb{R}^{d}$, while $\delta_{x_{0}}$ is the usual Dirac delta measure centered at $x_{0}$. For a given function $\rho \in L^{1}\left(\mathbb{R}^{d}\right)$ we use the notation

$$
\operatorname{supp}(\rho):=\left\{x \in \mathbb{R}^{d}: \int_{B(x, \epsilon)} \rho(x) d x \neq 0 \text { for all } \epsilon>0\right\} .
$$

We start with the observation that there exists a time-dependent mass-preserving scaling which removes the linear drift term $\partial_{v}(v f)$ from (1.1). More precisely, let us set

$$
\left\{\begin{array}{l}
f(v, \tau)=e^{d \tau} \rho(x, t) \\
x=e^{\tau} v \\
t=\frac{e^{d \gamma \tau}-1}{d \gamma} .
\end{array}\right.
$$

We obtain the following scaled equation for $\rho(x, t)$

$$
\partial_{t} \rho-\operatorname{div}\left(x \rho^{1+\gamma}\right)=0
$$

with $\rho(x, 0)=f_{I}(x)$. The new equation (2.2) has the great advantage of featuring a homogeneous nonlinearity in the drift term. We next write (2.2) in the 
non-conservative form

$$
\rho_{t}-(1+\gamma) \rho^{\gamma} x \cdot \nabla \rho=d \rho^{1+\gamma},
$$

which can only be done in case of smooth solutions. For a fixed $x_{0} \in \mathbb{R}^{d}$, the characteristic curves for the nonlinear first-order equation (2.3) starting at $x_{0}$ are denoted by $X_{x_{0}}(t)$, with $U_{x_{0}}(t):=\rho\left(X_{x_{0}}(t), t\right)$. They solve

$$
\begin{cases}\dot{X}_{x_{0}}(t)=-(1+\gamma) X_{x_{0}}(t) U_{x_{0}}(t)^{\gamma} & X_{x_{0}}(0)=x_{0} \\ \dot{U}_{x_{0}}(t)=d U_{x_{0}}^{1+\gamma}(t) & U_{x_{0}}(0)=u_{0}:=f_{I}\left(x_{0}\right) .\end{cases}
$$

The solution to the second equation in (2.4) is

$$
U_{x_{0}}(t)=\frac{f_{I}\left(x_{0}\right)}{\left(1-\gamma d f_{I}^{\gamma}\left(x_{0}\right) t\right)^{1 / \gamma}},
$$

which implies

$$
\left\{\begin{array}{l}
X_{x_{0}}(t)=x_{0}\left(1-\gamma d f_{I}^{\gamma}\left(x_{0}\right) t\right)^{(1+\gamma) / \gamma d} \\
\rho\left(X_{x_{0}}(t), t\right)=\frac{f_{I}\left(x_{0}\right)}{\left(1-\gamma d f_{I}^{\gamma}\left(x_{0}\right) t\right)^{1 / \gamma}}
\end{array}\right.
$$

The above formulae (2.5) suggest two important properties to hold for (2.2) in any dimension:

i) All solutions become unbounded in a finite time $t^{*}$ depending on the initial condition;

ii) The only point $x$ at which a solution may become unbounded is $x=0$.

Another property which is suggested by (2.5) is the confinement property

$$
\operatorname{supp}[\rho(\cdot, t)] \subset \operatorname{Conv}\left(\operatorname{supp}\left(f_{I}\right) \cup\{0\}\right) \quad \text { for all } t>0,
$$

which formally holds as long as solutions can be obtained via characteristics, since all the curves $X_{x_{0}}(\cdot)$ point towards the axis $x=0$. Here, $\operatorname{Conv}(A)$ denotes the convex hull of $A$. Finally, (2.5) suggests that $X_{0}(t) \equiv 0$ as long as the solution $\rho$ stays smooth.

As usual in the context of scalar conservation laws [8], we can use of the characteristic curves recovered in (2.5) to produce a local existence theorem for smooth solutions to (2.2) with smooth initial data. To perform this task, one has to invert the formula (2.5) for characteristics by recovering the initial point $x_{0}$ as a function of $X_{x_{0}}(t)$ and $t$. By the implicit function theorem, this can be done as long as the function

$$
\mathcal{F}\left(x, x_{0}, t\right):=x_{0}\left(1-\gamma d f_{I}^{\gamma}\left(x_{0}\right) t\right)^{(1+\gamma) / \gamma d}-x
$$

satisfies

$$
\frac{\mathrm{D} \mathcal{F}}{\mathrm{D} x_{0}} \neq 0
$$


A straightforward computation yields

$$
\begin{aligned}
\frac{\mathrm{D} \mathcal{F}}{\mathrm{D} x_{0}}= & \left(1-\gamma d f_{I}^{\gamma}\left(x_{0}\right) t\right)^{(1+\gamma) / \gamma d} \mathbb{I}_{d} \\
& -(1+\gamma) x_{0} \otimes \nabla f_{I}^{\gamma}\left(x_{0}\right)\left(1-\gamma d f_{I}^{\gamma}\left(x_{0}\right) t\right)^{(1+\gamma-\gamma d) / \gamma d} t .
\end{aligned}
$$

Since $\left.\frac{\partial \mathcal{F}}{\partial x_{0}}\right|_{t=0}=\mathbb{I}_{d}$, it is clear that characteristics do not cross at least on a small time strip provided $\nabla \rho_{0}^{\gamma}$ is finite almost everywhere. In one space dimension, the above condition (2.6) reads

$$
\left(1-\gamma f_{I}^{\gamma}\left(x_{0}\right) t\right)^{(1+\gamma) / \gamma}-(1+\gamma) x_{0}\left(1-\gamma f_{I}^{\gamma}\left(x_{0}\right) t\right)^{1 / \gamma}\left(f_{I}^{\gamma}\right)^{\prime}\left(x_{0}\right) t \neq 0,
$$

which is satisfied for all $0 \leq t<\left(\gamma f_{I}^{\gamma}\left(x_{0}\right)\right)^{-1}$ provided $x_{0} f_{I}^{\prime}\left(x_{0}\right) \leq 0$. Therefore, the latter condition ensures that the solution $\rho$ is classical on $(x, t) \in \mathbb{R} \times\left[0, t^{*}\right)$ with $t^{*}=\left(\gamma \max f_{I}^{\gamma}\right)^{-1}$. At $t=t^{*}, \rho$ tends to $+\infty$ at the point $x=0$.

In more than one space dimension, and for a radially symmetric initial datum $f_{I}\left(x_{0}\right)=\widetilde{f}_{I}\left(\left|x_{0}\right|\right),(2.6)$ reads

$$
\begin{aligned}
\frac{\mathrm{D} \mathcal{F}}{\mathrm{D} x_{0}}= & \left(1-\gamma d \tilde{f}_{I}^{\gamma}\left(\left|x_{0}\right|\right) t\right)^{(1+\gamma) / \gamma d} \mathbb{I}_{d} \\
& -(1+\gamma) x_{0} \otimes x_{0} \frac{\left(\tilde{f}_{I}^{\gamma}\right)_{r}\left(\left|x_{0}\right|\right)}{\left|x_{0}\right|}\left(1-\gamma d \tilde{f}_{I}^{\gamma}\left(\left|x_{0}\right|\right) t\right)^{(1+\gamma-\gamma d) / \gamma d} t .
\end{aligned}
$$

Therefore, in case $\rho$ is initially radially non-increasing, the solution is classical until $t=\left(\gamma d \max f_{I}^{\gamma}\right)^{-1}$. This is due to the fact that the matrix $x_{0} \otimes x_{0}$ is non-negative definite. We have therefore proved the following:

Theorem 2.1 (Local existence of smooth solutions). Let $f_{I} \in C^{1} \cap L^{\infty}\left(\mathbb{R}^{d}\right)$, with $f_{I} \geq 0$. Then there exist a time $t^{*}>0$ and $\rho(\cdot, \cdot) \in C^{1}\left(\left[0, t^{*}\right) \times \mathbb{R}^{d}\right)$ solution to (2.3). Moreover:

- If $f_{I}$ is radially non increasing, then the maximal time $t^{*}$ is given by

$$
t^{*}=\left(\gamma d \max f_{I}^{\gamma}\right)^{-1}
$$

- If the maximal time is given by (2.7), then the solution $\rho$ blows up at $x=0$, namely

$$
\lim _{(x, t) \rightarrow\left(0, t^{*}\right)} \rho(x, t)=+\infty
$$

- If the maximal time satisfies $t^{*}<\left(\gamma d \max f_{I}^{\gamma}\right)^{-1}$, then the solution $\rho$ develops a discontinuity before blowing up.

The result in Theorem 2.1 shows that discontinuities may arise in a finite time, as usual in this context, since characteristics may cross in finite time. The result in Theorem 2.1 can be easily re-formulated in terms of the original equation (1.1). We omit the details. 


\section{The one-dimensional case}

Let us then recover a reasonable notion of weak solution at least in the one-dimensional case. Being the only possible concentration point at zero, the characteristics all pointing towards the origin, and dealing with a conservation law, we cannot expect anything else than a concentration of mass at zero. Then, we need a notion of measure solution that allows for this possibility. We are going to start by introducing a notion of weak solution that avoids the concentration at the origin issue by allowing a loss of mass through the origin. This will be just an intermediate step in our construction.

Definition 3.1 (Weak solutions). Let $T>0$. Then, $\rho(x, t)$ is a weak solution to (2.2) on $[0, T]$ with initial datum $f_{I} \in L^{\infty}(\mathbb{R})$ if and only if

- $x \rho^{1+\gamma} \in L^{\infty}(\mathbb{R} \times[0, T))$, and

- for all $\varphi \in C_{c}^{\infty}(\mathbb{R} \times[0, T))$ such that $\varphi(0)=0$, one has

$$
\begin{aligned}
\int_{\mathbb{R}^{d}} \int_{0}^{T} \varphi_{t}(x, t) \rho(x, t) d x d t= & \int_{\mathbb{R}^{d}} \varphi(x, 0) f_{I}(x) d x \\
& +\int_{\mathbb{R}^{d}} \int_{0}^{T} \rho^{1+\gamma}(x, t) x \cdot \nabla \varphi(x, t) d x d t .
\end{aligned}
$$

We will clarify in Section 3 that examples of non-uniqueness of weak solutions can be easily found. Therefore a definition of entropy solution in the spirit of [21] is needed. This notion can be written for the evolution equation (2.2) but we prefer to postpone it since it will be much clearer through a new change of variables.

In order to deal with entropy solutions, we shall restrict to the case of initial condition $f_{I} \in B V(\mathbb{R})$. Note that in one dimension, this implies that $f_{I} \in L^{1} \cap$ $L^{\infty}(\mathbb{R})$. We will assume that we work with probability measures denoted by $\mathcal{P}(\mathbb{R})$, and thus with unit mass non-negative densities. Further restrictions will be required later on.

Let $f_{I} \in \mathcal{P}(\mathbb{R}) \cap B V(\mathbb{R})$ with the additional condition $f_{I} \in C^{1}(\mathbb{R})$, and let $\rho$ be the local-in-time $C^{1}$ bounded solution to

$$
\rho_{t}-\left(x \rho^{1+\gamma}\right)_{x}=0
$$

with initial datum $f_{I}$ provided by Theorem 2.1. As we already pointed out in Section 2, the characteristic curve generated at $x_{0}=0$ is a vertical line, and it therefore separates the mass on $x<0$ from the mass on $x>0$. More precisely, let $t^{*}$ be the maximal time of existence of $\rho$ provided in Theorem 2.1, for all $t \in\left[0, t^{*}[\right.$ we have

$$
\begin{aligned}
& \int_{-\infty}^{0} \rho(x, t) d x=m_{L}:=\int_{-\infty}^{0} f_{I}(x) d x, \\
& \int_{0}^{+\infty} \rho(x, t) d x=m_{R}:=\int_{0}^{+\infty} f_{I}(x) d x .
\end{aligned}
$$


This property is due to the expression for the flux $x \rho^{1+\gamma}$, which clearly vanishes at $x=0$ as long as $\rho$ is bounded. We introduce the scaled solution $u$ as follows:

$$
\begin{aligned}
& u(\xi, t)=x^{\prime}(\xi) \rho(x(\xi), t), \quad \xi \in \mathbb{R}, \quad t \geq 0 \\
& x(\xi)=\operatorname{sign}(\xi) \frac{1}{1+\gamma}(\gamma|\xi|)^{(1+\gamma) / \gamma}, \quad x^{\prime}(\xi)=(\gamma|\xi|)^{1 / \gamma} .
\end{aligned}
$$

It is immediately checked that

$$
\begin{aligned}
& \int_{0}^{+\infty} \rho(x, t) d x=\int_{0}^{+\infty} u(\xi, t) d \xi \\
& \int_{-\infty}^{0} \rho(x, t) d x=\int_{-\infty}^{0} u(\xi, t) d \xi
\end{aligned}
$$

and that $u$ satisfies

$$
u_{t}-\operatorname{sign}(\xi)\left(\frac{1}{1+\gamma} u^{1+\gamma}\right)_{\xi}=0 .
$$

Since $\rho$ is bounded, $u$ has the trace property

$$
u(0, t)=0 \quad \text { for all } t \in\left[0, t^{*}[.\right.
$$

The change of variable $x=x(\xi)$ introduced in (3.3) admits the inverse transformation

$$
\xi(x)=\operatorname{sign}(x) \frac{1}{\gamma}[(1+\gamma)|x|]^{\frac{\gamma}{1+\gamma}},
$$

which is not differentiable at $x=0$. However, in view of (3.5), the inverse change of variable

$$
\rho(x, t)=\xi^{\prime}(x) u(\xi(x), t)
$$

is well-defined for all $x \neq 0$.

Although the change of variable (3.3) has the advantage of being defined on $\xi \in \mathbb{R}$, the equation (3.4) may be uncomfortable to deal with because of the discontinuous coefficient at $\xi=0$. We shall therefore consider two separate Cauchy problems:

$$
\begin{aligned}
& \begin{cases}u_{t}-\left(\frac{1}{1+\gamma} u^{1+\gamma}\right)_{\xi}=0 & \xi>0, \quad t \geq 0 \\
u(\xi, 0)=u_{I, R}(\xi):=(\gamma \xi)^{1 / \gamma} f_{I}\left(\frac{(\gamma \xi)^{(1+\gamma) / \gamma}}{1+\gamma}\right) & \xi>0,\end{cases} \\
& \begin{cases}u_{t}+\left(\frac{1}{1+\gamma} u^{1+\gamma}\right)_{\xi}=0 & \xi<0, \quad t \geq 0 \\
u(\xi, 0)=u_{I, L}(\xi):=(-\gamma \xi)^{1 / \gamma} f_{I}\left(-\frac{(-\gamma \xi)^{(1+\gamma) / \gamma}}{1+\gamma}\right) & \xi<0 .\end{cases}
\end{aligned}
$$


We shall denote solutions to (3.8) (to (3.9) respectively) by $u_{R}$ ( $u_{L}$ respectively), and we shall often refer to $u$ as

$$
u(\xi, t)= \begin{cases}u_{L}(\xi, t) & \xi<0 \\ u_{R}(\xi, t) & \xi>0\end{cases}
$$

As the initial condition in both problems (3.8) and (3.9) is non-negative, the characteristic curves generated near the boundary $\xi=0$ are outgoing. Therefore, no boundary condition needs to be prescribed in order to achieve a unique entropy solution for the initial value problems (3.8)-(3.9), see [1,9]. Moreover, it is well known in the context of scalar conservation laws with uniformly convex or concave flux that the classical notion of entropy solution in Kružkov sense [21] is equivalent to the notion of weak solutions together with the Oleinik's condition [24]. This condition roughly speaking requires a one-sided bound for the space derivative in a distributional sense. In the present context, since the convexity of the flux depends on the sign of $\xi$, and in view of well known results $c f$. [15], the correct Oleinik type condition is given by the distributional inequality

$$
-\operatorname{sign}(\xi)\left(u^{\gamma}\right)_{\xi} \leq \frac{C}{t}, \quad \xi \neq 0, \quad t>0
$$

for some positive constant $C$. However, our assumption that $f_{I} \in B V(\mathbb{R})$ allows us to formulate the entropy condition in the even simpler way

$$
\begin{array}{ll}
\lim _{\xi \nearrow \xi_{0}^{-}} u(\xi, t) \geq \lim _{\xi \searrow \xi_{0}^{+}} u(\xi, t) & \text { if } \xi_{0}<0, \\
\lim _{\xi \nearrow \xi_{0}^{-}} u(\xi, t) \leq \lim _{\xi \searrow \xi_{0}^{+}} u(\xi, t) & \text { if } \xi_{0}>0 .
\end{array}
$$

Definition 3.2. Let $f_{I} \in \mathcal{P}(\mathbb{R}) \cap B V(\mathbb{R})$. A function $u \in L^{\infty}\left([0,+\infty) ; L^{1}(\mathbb{R}) \cap\right.$ $B V(\mathbb{R})$ ) is an entropy solution to the Cauchy problem (3.8)-(3.9) if $u$ can be written as (3.10) with $u_{R}$ solving (3.8) and $u_{L}$ solving (3.9) in a weak sense, and $u$ satisfies the jump conditions (3.11).

We can therefore collect the results in $[1,15,21,24]$ adapted to problem (3.8)(3.9) in the following:

Theorem 3.3. Let $f_{I} \in \mathcal{P}(\mathbb{R}) \cap B V(\mathbb{R})$, then there exists a unique $u \in L^{\infty}([0,+\infty)$; $L^{1}(\mathbb{R}) \cap B V(\mathbb{R})$ ) entropy solution to the problem (3.8)-(3.9) in the sense of Definition 3.2 .

Remark 3.4 (Comparison principle). As a consequence of the classical theory of scalar conservation laws (see [8, Chapter 6]), for two given entropy solutions $u_{L}^{1}, u_{L}^{2}$ to (3.8) we have $u_{L}^{1}(x, t) \leq u_{L}^{2}(x, t)$ for almost every $x<0$ and for all $t>0$ provided $u_{L}^{1}(x, 0) \leq u_{L}^{2}(x, 0)$. 
Remark 3.5 (Mass conservation for solutions to (2.2)). It can be easily checked (see the proof of Theorem 5.2 below) that if $u$ is a entropy solution to (3.8)-(3.9) then $\rho$ defined from $u$ via the scaling (3.7) is a weak solution to $(2.2)$ on $[0,+\infty]$ according to Definition 3.1.

If the two traces $u\left(0^{-}, t\right)$ and $u\left(0^{+}, t\right)$ of the entropy solutions to (3.8)-(3.9) are zero, the total mass of the system is conserved. However, this property is lost as soon as one of the two traces becomes positive. In this case, the solution $\rho$ to (3.2) becomes unbounded at $x=0$ due to the change of variables (3.7) and start losing mass at the origin. Our conjecture is that, speaking in terms of $\rho$, such lost mass gets concentrated to a delta measure at the origin, as we shall see in the example below. Therefore, the notion of solution $u$ provided in Definition 3.2 will be used to extend our solutions $\rho$ in a measure sense after their blow up in a finite time.

Example 3.6 (An explicit solution). We shall provide an explicit solution to (3.2) by setting as initial datum

$$
\rho(x, 0)=f_{I}(x)= \begin{cases}1 & \text { if } 0 \leq x \leq \frac{1}{1+\gamma} \\ 0 & \text { otherwise }\end{cases}
$$

We use the scaling (3.3), which turns the initial condition $f_{I}$ into

$$
u_{0}(\xi):=(\gamma \xi)^{1 / \gamma} f_{I}\left(\frac{1}{1+\gamma}(\gamma|\xi|)^{(1+\gamma) / \gamma}\right)=(\gamma \xi)^{1 / \gamma} \chi_{[0,1 / \gamma]}
$$

We recall the characteristic equations for the scaled equation (3.8):

$$
\left\{\begin{array}{l}
\xi_{\xi_{0}}(t)=\xi_{0}(1-\gamma t) \\
u\left(\xi_{\xi_{0}}(t), t\right)=u_{0}\left(\xi_{0}\right)
\end{array}\right.
$$

for $0 \leq \xi_{0} \leq 1 / \gamma$. If $\xi_{0}>1 / \gamma$ we have $\xi_{\xi_{0}}(t) \equiv \xi_{0}$. There is a rarefaction fan $1 / \gamma-t<\xi<1 / \gamma$ which is filled up with the characteristic lines $\xi_{\lambda}(t)=\frac{1}{\gamma}-\lambda t$, with $\lambda \in(0,1)$. The profile in the rarefaction fan is

$$
u(\xi, t)=\left(\frac{1-\gamma \xi}{\gamma t}\right)^{1 / \gamma}
$$

Therefore, the unique entropy solution to (3.8) with initial datum $u_{0}$ is

$$
u(\xi, t)= \begin{cases}\left(\frac{\gamma \xi}{1-\gamma t}\right)^{1 / \gamma} & \text { if } 0 \leq \xi \leq \frac{1}{\gamma}-t \text { and } 0 \leq t<\frac{1}{\gamma} \\ \left(\frac{1-\gamma \xi}{\gamma t}\right)^{1 / \gamma} & \text { if } \max \left\{0, \frac{1}{\gamma}-t\right\} \leq \xi \leq \frac{1}{\gamma} \\ 0 & \text { otherwise. }\end{cases}
$$


By applying the scaling (3.7), we obtain the solution $\rho$ to (3.2) with initial datum $f_{I}$ given in (3.12)

$$
\rho(x, t)= \begin{cases}\left(\frac{1}{1-\gamma t}\right)^{\frac{1}{\gamma}} & \text { if } 0 \leq x \leq \frac{(1-\gamma t)^{\frac{\gamma}{1+\gamma}}}{1+\gamma} \text { and } 0 \leq t \leq 1 / \gamma \\ {\left[\frac{((1+\gamma) x)^{-\frac{\gamma}{1+\gamma}}-1}{\gamma t}\right]^{\frac{1}{\gamma}}} & \text { if } \max \left\{0, \frac{(1-\gamma t)^{\frac{\gamma}{1+\gamma}}}{1+\gamma}\right\} \leq x \leq \frac{1}{1+\gamma} \\ 0 & \text { otherwise. }\end{cases}
$$

It is easily seen that $\rho$ in (3.13) preserves the total mass $\int_{0}^{+\infty} \rho(x, t) d x=\frac{1}{1+\gamma}$ as long as $t \leq \frac{1}{\gamma}$. At $t=t^{*}:=\frac{1}{\gamma}$, the total mass starts decreasing. More precisely, for $t \geq t^{*}$

$$
m(t):=\int_{0}^{+\infty} \rho(x, t) d x=\left(\frac{1}{\gamma t}\right)^{1 / \gamma} \frac{1}{1+\gamma} .
$$

Such a behaviour suggests that the mass of $\rho(\cdot, t)$ starts concentrating at $t>t^{*}$, in particular that a Dirac's delta part centered at zero with mass

$$
\frac{1}{1+\gamma}\left(1-\left(\frac{1}{\gamma t}\right)^{1 / \gamma}\right)
$$

is generated for $t>\frac{1}{\gamma}$. Please notice that the mass concentrated at zero is continuous in time, and it converges to the total mass of $\rho$ as $t \rightarrow+\infty$. The solution found here can be easily scaled back to a solution $f$ to (1.1) via the scaling (2.1). The qualitative behaviour is substantially the same, with the only difference that the $f$ is subject to a confinement, which makes the support of $f$ shrink to $v=0$ for large times with algebraic rate.

Remark 3.7 (Need for a measure solution theory). The above example shows that the entropy solution concept provided in Definition 3.1 is only satisfactory for short times, and it therefore needs to be improved to include also measure solutions. On the other hand, allowing for a Dirac's delta solution in (3.2) is complicated in view of the nonlinearity w.r.t. $\rho$ in the drift term. In the next section we shall solve this issue by producing a theory for measure solutions. In particular, the concentration phenomenon (which is somewhat hidden in the above computation) will be made evident.

\section{Measure solutions}

Example 3.6 shows that a notion of measure solution is needed in order to establish a global-in-time existence theory for (3.2). In this section we shall perform this task by considering the equation satisfied by the pseudo-inverse of the distribution function of the solution to (3.2). Let us start by providing a formal argument to 
justify the use of the pseudo inverse equation. Assume $\rho$ to be the unique shorttime smooth solution to (3.2), defined on $\mathbb{R} \times[0, T]$, with initial datum $f_{I}$ having unit mass, and set

$$
F(x, t):=\int_{-\infty}^{x} \rho(\xi, t) d \xi .
$$

Let $[0,1] \times[0, T] \ni(z, t) \mapsto X(z, t) \in \mathbb{R}$ be the pseudo-inverse of $F$

$$
X(z, t)=\inf \{x: F(x, t)>z\} .
$$

Formally, $X$ satisfies the equation

$$
X_{t}=-\frac{X}{X_{z}^{\gamma}} .
$$

The interplay between (4.1) and (3.2) will be more clear in the proof of Theorem 5.2. Clearly, the equation (4.1) in the above form does not allow to consider a possible solution $X(z, t)$ which is constant on some interval $I \subset[0,1]$. Let us then instead consider the more general version

$$
X_{t}\left|X_{z}\right|^{\gamma}+X=0
$$

in which we have allowed for possible changes in the sign of $X_{z}$. Please notice that having $X(\cdot, t)$ constant on some interval $I \subset[0,1]$ is equivalent to a jump discontinuity for $F(\cdot, t)$ at some point $x_{0}$, which means that $\rho=\partial_{x} F$ is a probability measure with a non trivial Dirac delta part at $x_{0}$. As already pointed out before in Section 2, we shall see that solutions to (3.2) can only concentrate at $x=0$.

Remark 4.1 (Connected components of $\rho$ ). Let us also mention that the equation (4.2) loses its meaning if the solution $\rho(\cdot, t)$ to (3.2) is supported on a disconnected set. Suppose for instance that the curve $x=x_{1}(t)$ is the left edge of a connected component of $\rho(\cdot, t)$ with $x_{1}(t)<\sup \operatorname{supp}(\rho)$, and assume for simplicity that no concentration occurs. Then, $X(\cdot, t)$ will be discontinuous at the point $z_{1}=$ $\int_{-\infty}^{x_{1}(t)} \rho(x, t) d x$ (which is 'locally' constant in time). In case of a finite number of connected components, $X$ would be discontinuous on a set of stationary points $z_{1}, \ldots, z_{k}$, until two connected components possibly merge into one which means that $X$ becomes continuous on one of the $z_{j}$ 's. On the other hand, this creates unnecessary complications to the notation and the calculations below. Therefore, we shall restrict for simplicity to the case of an initial condition $f_{I}$ with a single connected component in its support, for more details see Section 5.

Equation (4.2) can be partially solved via the method of characteristics for fully nonlinear first order equations, see e.g. [23]. Let us recall that (4.2) is coupled with the initial condition

$$
X(z, 0)=\bar{X}(z):=\inf \left\{x: \int_{-\infty}^{x} f_{I}(y) d y>z\right\},
$$

where $z \in[0,1]$. We shall assume for simplicity that $\bar{X} \in \mathcal{X}_{0} \cap C^{1}((0,1))$. 
Let us then define characteristics as follows: the vector $Y(s):=(z(s), t(s))$ is the characteristic curve on the set $[0,1] \times[0, T]$. We shall start by considering characteristics generated at a point $\left(z_{0}, 0\right)$ in the initial interval $t=0$. The solution evaluated on the characteristics is $U(s):=X(Y(s))=X(z(s), t(s))$. The gradient of $X$ along the characteristics is given by $P(s):=\left(p_{1}(s), p_{2}(s)\right):=$ $\left(X_{z}(Y(s)), X_{t}(Y(s))\right)$. As for the initial conditions at $s=0$, for a fixed initial point $z_{0} \in[0,1]$ we have

$$
\begin{aligned}
& Y(0)=\left(z_{0}, 0\right), \quad U(0)=\bar{X}\left(z_{0}\right) \\
& P(0)=\left(p_{1}(0), p_{2}(0)\right)=\left(\bar{X}_{z}\left(z_{0}\right),-\bar{X}\left(z_{0}\right)\left|\bar{X}_{z}\left(z_{0}\right)\right|^{-\gamma}\right) .
\end{aligned}
$$

Let us differentiate (4.2) with respect to $z$ first and $t$ second:

$$
\begin{aligned}
& X_{t z}\left|X_{z}\right|^{\gamma}+\gamma \operatorname{sign}\left(X_{z}\right) X_{t}\left|X_{z}\right|^{\gamma-1} X_{z z}+X_{z}=0 \\
& X_{t t}\left|X_{z}\right|^{\gamma}+\gamma \operatorname{sign}\left(X_{z}\right) X_{t}\left|X_{z}\right|^{\gamma-1} X_{z t}+X_{t}=0 .
\end{aligned}
$$

We have

$$
\begin{aligned}
& \dot{p}_{1}(s)=X_{z z}(Y(s)) \dot{z}(s)+X_{z t}(Y(s)) \dot{t}(s) \\
& \dot{p}_{2}(s)=X_{t z}(Y(s)) \dot{z}(s)+X_{t t}(Y(s)) \dot{t}(s) .
\end{aligned}
$$

In order to remove the second derivatives in (4.5) and (4.6) we require

$$
\begin{aligned}
\dot{z}(s) & =\gamma \operatorname{sign}\left(X_{z}(Y(s))\right) X_{t}(Y(s))\left|X_{z}(Y(s))\right|^{\gamma-1} \\
& =\gamma \operatorname{sign}\left(p_{1}(s)\right)\left|p_{1}(s)\right|^{\gamma-1} p_{2}(s) \\
\dot{t}(s) & \left.=\mid X_{z}(Y(s))\right)\left.\right|^{\gamma}=\left|p_{1}(s)\right|^{\gamma}
\end{aligned}
$$

so that (4.3) and (4.4) imply

$$
\dot{p}_{1}(s)=-p_{1}(s), \quad \dot{p}_{2}(s)=-p_{2}(s) .
$$

Finally, differentiating $U(s)=X(z(s), t(s))$ with respect to $s$ yields

$$
\dot{U}(s)=p_{1}(s) \dot{z}(s)+p_{2}(s) \dot{t}(s)=(\gamma+1)\left|p_{1}(s)\right|^{\gamma} p_{2}(s) .
$$

Solving (4.9) gives

$$
p_{1}(s)=\bar{X}_{z}\left(z_{0}\right) e^{-s}>0, \quad p_{2}(s)=-\bar{X}\left(z_{0}\right) \bar{X}_{z}\left(z_{0}\right)^{-\gamma} e^{-s},
$$

and therefore (4.10) gives $\dot{U}(s)=(\gamma+1) \bar{X}\left(z_{0}\right) e^{-(1+\gamma) s}$, which yields $U(s)=$ $\bar{X}\left(z_{0}\right) e^{-(1+\gamma) s}$. Finally, solving (4.7) and (4.8) yields

$$
\dot{z}(s)=-\gamma \bar{X}\left(z_{0}\right) \bar{X}_{z}\left(z_{0}\right)^{-1} e^{-\gamma s}, \quad \dot{t}(s)=\bar{X}_{z}\left(z_{0}\right)^{\gamma} e^{-\gamma s},
$$


which result in

$$
z(s)=z_{0}-\bar{X}\left(z_{0}\right) \bar{X}_{z}\left(z_{0}\right)^{-1}\left(1-e^{-\gamma s}\right) \quad t(s)=\frac{1}{\gamma} \bar{X}_{z}\left(z_{0}\right)^{\gamma}\left(1-e^{-\gamma s}\right) .
$$

We notice that $z$ can be written as a function of $t$ as follows:

$$
z=z(t)=z_{0}-\gamma \bar{X}\left(z_{0}\right) \bar{X}_{z}\left(z_{0}\right)^{-(\gamma+1)} t .
$$

Example 4.2 (Explicit solution revisited). Let us take as initial condition $\bar{X}(z)=$ $z$, which corresponds (in terms of probability densities $\rho$ ) to the initial condition in Example 3.6 up to a dilation and a multiplication by a constant. The characteristic curves obtained above are given by

$$
\begin{aligned}
& U(s)=z_{0} e^{-(1+\gamma) s}, \quad z(s)=z_{0} e^{-\gamma s}, \quad t(s)=\frac{1-e^{-\gamma s}}{\gamma} \\
& p_{1}(s)=e^{-s}, \quad p_{2}(s)=-z_{0} e^{-s} .
\end{aligned}
$$

Characteristics can be also written as

$$
z=\tilde{z}(t)=z_{0}(1-\gamma t), \quad U=\tilde{U}(t)=z_{0}(1-\gamma t)^{\frac{1+\gamma}{\gamma}} .
$$

The above characteristics occupy the region $A=\left\{(z, t) \in[0,1] \times \mathbb{R}_{+}: 0 \leq z \leq\right.$ $1-\gamma t\}$. The formula for the solution $X$ in $A$ is

$$
X(z, t)=z(1-\gamma t)^{1 / \gamma} .
$$

In order to fill up the region $B:=[0,1] \times \mathbb{R}_{+} \backslash A$, we introduce the rarefaction wave originated at the point $\left(z_{0}, t_{0}\right)=(1,0)$ in the next computations. Since $U(0)=1$, assuming that the equation (4.2) is satisfied on the region $B$, we have the following set of characteristics:

$$
\begin{aligned}
& U(s)=e^{-(\gamma+1) s} \\
& z(s)=1+\lambda^{-1}\left(e^{-\gamma s}-1\right) \\
& t(s)=\frac{\lambda^{\gamma}}{\gamma}\left(1-e^{-\gamma s}\right) \\
& p_{1}(s)=\lambda e^{-s}, \quad p_{2}(s)=-\lambda^{-\gamma} e^{-s},
\end{aligned}
$$

where $\lambda=p_{1}(0)$. Unlike in the region $A$, the value $\lambda$ should not be prescribed according to the initial condition, since the characteristic lines we are computing are originated at $(1,0)$ and enter the region $B$. Therefore, heuristically speaking we are not interested in the slope of the initial condition at $z=1$, since the above mentioned characteristic lines 'will not touch' the profile originated from the initial condition. In order to fill the whole region $B$, the parameter $\lambda$ should be taken in the set $(1,+\infty)$. Such parameter can be seen as the analogous of a self-similar 
variable. In order to find the explicit formula for $X=X(z, t)$ on $B$, we use (4.13) and (4.14) to write $\lambda$ and $s$ in terms of $z$ and $t$. Inserting (4.14) in (4.12) gives

$$
U(s)=\tilde{U}(t)=\left(1-\gamma \lambda^{-\gamma} t\right)_{+}^{\gamma+1} \gamma .
$$

The positive part is needed in order to make formula (4.15) well-defined for all $t$. Now (4.13) provides the formula for $\lambda$, namely $\lambda=\left(\frac{\gamma t}{1-z}\right)^{\frac{1}{1+\gamma}}$, which can be inserted in (4.15) to get to the formula for $X=X(z, t)$ on the region $B$ :

$$
X(z, t)=\left[1-(\gamma t)^{\frac{1}{1+\gamma}}(1-z)^{\frac{\gamma}{1+\gamma}}\right]_{+}^{\frac{1+\gamma}{\gamma}} .
$$

From (4.16) and (4.11) we deduce the following properties:

- When $t<1 / \gamma$, the two profiles for $X$ on $A$ and $B$ match in a $C^{1}$ way at the point $z=1-\gamma t$, with derivative equals to $\left.\frac{\partial X}{\partial z}\right|_{z=1-\gamma t}=(1-\gamma t)^{1 / \gamma}$;

- When $t \geq 1 / \gamma$, only the profile for $X$ on $B$ survives. $X$ is constant zero on the interval $z \in\left[0,1-(\gamma t)^{-1 / \gamma}\right]$, and $X(\cdot, t) \in C^{1}[0,1)$ for all $t \geq 1 / \gamma$;

- $X$ has an infinite $z$-derivative on $z=1$ for all times $t>0$.

Let $\mu(t) \in \mathcal{P}(\mathbb{R})$ the distributional $x$-derivative of $F(\cdot, t)$ the pseudo inverse of $X(\cdot, t)$. It is easily seen that $\mu(t)=\left[1-(\gamma t)^{-1 / \gamma}\right] \delta_{0}+\rho(\cdot, t)$ with $\rho(\cdot, t) \in$ $B V(\mathbb{R})$ being the weak solution found in Example 3.6 except suitable time change of variables to have unit mass. In particular, as $t \rightarrow+\infty$, the concentrated mass of $\mu(t)$ tends to the total mass 1 as in the Example 3.6. Moreover, the concentrated mass is continuous in time.

We now want to recover an equivalent formulation of the Oleinik condition (3.11) in terms of the pseudo inverse variable $X$. Let $f_{I} \in \mathcal{M}_{0}$ and let $\rho$ be the local-in-time solution to (3.2) provided by Theorem 2.1 on $0 \leq t<t^{*}$. Let $u_{R}$ solve (3.8) and $u_{L}$ solve (3.9) respectively, and let $u$ be defined as in (3.10). Notice that $\rho$ and $u$ are linked through the scaling (3.7). As $\rho$ is bounded on $0 \leq t<t^{*}$, then $u(0, t)=0$ for all $0 \leq t<t^{*}$. Denote

$$
m_{L}=\int_{-\infty}^{0} u(\xi, t) d \xi, \quad m_{R}=1-m_{L}=\int_{0}^{+\infty} u(\xi, t) d \xi .
$$

In view of $u(0, t)=0$, it is obvious that $m_{L}$ and $m_{R}$ are constant in time on $0 \leq$ $t<t^{*}$. Let us define the primitive variables

$$
\begin{aligned}
& G_{L}(\xi, t)=\int_{0}^{\xi} u_{L}(\eta, t) d \eta, \quad \xi>0, \\
& G_{R}(\xi, t)=\int_{-\infty}^{\xi} u_{R}(\eta, t) d \eta \quad \xi<0,
\end{aligned}
$$


and their pseudo-inverses

$$
\begin{aligned}
& \left.\left.Y_{R}(z, t)=\inf \left\{\xi>0: m_{L}+G_{R}(\xi, t)>z\right\}, \quad z \in\right] m_{L}, 1\right], \\
& Y_{L}(z, t)=\inf \left\{\xi<0: G_{L}(\xi, t)>z\right\}, \quad z \in\left[0, m_{L}[,\right. \\
& Y(z, t)= \begin{cases}Y_{L}(z, t) & z \in\left[0, m_{L}[\right. \\
Y_{R}(z, t) & z \in\left[m_{L}, 1\right] .\end{cases}
\end{aligned}
$$

Since supp $[u]$ is a connected interval and $u \in L^{\infty}(\mathbb{R})$, then both $Y_{R}$ and $Y_{L}$ are continuously differentiable in their respective domains with the possible exceptions of the boundary points $z=0$ and $z=1$ (for instance, $Y_{R}$ has an infinite derivative at $z=1$ in case $\sup \left(\operatorname{supp}\left[u_{R}\right]\right)>0$, since $u_{R}$ cannot have decreasing shocks on $\xi>0$, and it therefore tends to zero at the right edge of its support).

Analogously to the $u$ variable, let us introduce left and right distribution functions and pseudo-inverses for $\rho$. More precisely, let

$$
\begin{aligned}
& F_{R}(x, t)=\int_{0}^{x} \rho(\eta, t) d \eta \quad x>0 \\
& F_{L}(x, t)=\int_{-\infty}^{x} \rho(\eta, t) d \eta \quad x<0 \\
& \left.\left.X_{R}(z, t)=\inf \left\{x: m_{L}+F_{R}(x, t)>z\right\} \quad z \in\right] m_{L}, 1\right] \\
& X_{L}(z, t)=\inf \left\{x: F_{L}(x, t)>z\right\} \quad z \in\left[0, m_{L}[\right. \\
& X(z, t)= \begin{cases}X_{L}(z, t) & z \in\left[0, m_{L}[\right. \\
X_{R}(z, t) & \left.z \in] m_{L}, 1\right] .\end{cases}
\end{aligned}
$$

By means of the scaling (3.7), a straightforward computation yields

$$
Y(z, t)=\frac{\operatorname{sign}(X(z, t))}{\gamma}((1+\gamma)|X(z, t)|)^{\frac{\gamma}{1+\gamma}} .
$$

Since the boundary condition $u_{L}(0, t)=u_{R}(0, t)=0$ is satisfied, $Y$ solves the equation

$$
Y_{t} Y_{z}^{\gamma}+\operatorname{sign}(Y) \frac{1}{1+\gamma}=0, \quad z \in[0,1], t>0 .
$$

We are now ready to rephrase the jump admissibility conditions (3.11) in terms of the pseudo inverse variable $X$ defined in (4.19). Assume for instance that $X_{z}(\cdot, t)$ has an increasing jump on some point $z_{0} \in(0,1)$ with $X\left(z_{0}, t\right)>0$. Then, $Y(\cdot, t)$ defined in (4.20) will have an increasing jump at the same point $z_{0}$. Formally, since $G_{R}$ is the pseudo-inverse of $Y$ on the set $Y>0$, this means that $G_{R}$ has a decreasing jump in its derivative, i.e. $u_{R}$ has an decreasing jump, which is not admissible due to (3.11). Thanks to this argument, and with a specular one for the case $X\left(z_{0}, t\right)<0$, we can rephrase condition (3.11) as follows: 
(XJ) Assume $X(\cdot, t)$ has a jump discontinuity in its $z$-derivative $X_{z}(\cdot, t)$ at some point $z_{0}$. Then

$$
\begin{array}{ll}
\lim _{z \nearrow z_{0}^{-}} X_{z}(z, t)<\lim _{z \searrow z_{0}^{+}} X_{z}(z, t) & \text { if } X\left(z_{0}, t\right)<0 \\
\lim _{z \nearrow z_{0}^{-}} X_{z}(z, t)>\lim _{z \searrow z_{0}^{+}} X_{z}(z, t) & \text { if } X\left(z_{0}, t\right)>0 .
\end{array}
$$

\section{Existence and uniqueness of entropy measure solutions}

We are now ready to state our notion of measure solution to (4.2). We shall use the following notation:

$$
\mathcal{M}_{0}=\left\{\mu \in \mathcal{P}(\mathbb{R}): \mu=m \delta_{0}+\rho \mathcal{L}_{1}, m \in[0,1], \rho \in L_{+}^{1}(\mathbb{R}), \operatorname{supp}(\rho)=[a, b], a, b \in \mathbb{R}\right\} .
$$

For a given $\mu \in \mathcal{M}_{0}$, with $\mu=m \delta_{0}+\rho \mathcal{L}_{1}$ the pseudo-inverse distribution

$$
X_{\mu}(z)=\inf \{x \in \mathbb{R}: \mu((-\infty, x])>z\}
$$

satisfies meas $\left(\left\{X_{\mu}(z)=0\right\}\right)=m$, and the following properties:

(X1) $X_{\mu}$ is continuous on $[0,1]$,

(X2) $X_{\mu}$ is non-decreasing on $[0,1]$.

We shall use the notation

$$
\mathcal{X}_{0}=\left\{X \in L^{\infty}([0,1]): \quad X \text { satisfies }(\mathrm{X} 1)-(\mathrm{X} 2)\right\} .
$$

For future use, we also need the additional properties

(X3) If $X_{\mu}(z) \neq 0$, then $X_{\mu}$ has finite nonzero left and right derivatives $\partial_{z}^{-} X_{\mu}(z)$ and $\partial_{z}^{+} X_{\mu}(z)$,

(X4) If $X_{\mu}(0)<0$, then $\lim _{h \backslash 0} \frac{X_{\mu}(h)-X_{\mu}(0)}{h}=+\infty$,

(X5) If $X_{\mu}(1)>0$, then $\lim _{h \backslash 0} \frac{X_{\mu}(1)-X_{\mu}(1-h)}{h}=+\infty$

and the notation

$$
\mathcal{X}_{0}^{+}=\left\{X \in \mathcal{X}_{0}: \quad X \text { satisfies (X3)-(X5) }\right\} .
$$

Please notice that the conditions (X4) and (X5) are nothing but avoiding nonentropic jump discontinuities at the edges of the support of $\rho$. Condition (X3) will be used later on to highlight the fact that $\rho$ is in $B V_{l o c}(\mathbb{R} \backslash\{0\})$.

Definition 5.1 (Entropy measure solutions). Let $f_{I} \in \mathcal{P}(\mathbb{R}) \cap B V(\mathbb{R})$ with connected compact support. A curve of probability measures $[0,+\infty) \ni t \mapsto \mu(t) \in$ $\mathcal{M}_{0}$ is an entropy measure solution to (2.2) with initial datum $f_{I}$ if, given $F(x, t):=$ $\mu(t)((-\infty, x])$ its cumulative distribution, and the pseudo-inverse function $X(\cdot, t)$ : $[0,1] \rightarrow \mathbb{R}$ of $F(\cdot, t)$, the following properties are satisfied: 
(In) $\mu(0)=f_{I} \mathcal{L}_{1}$,

(Re) $X(\cdot, t) \in \mathcal{X}_{0}^{+}$for all times $t \geq 0$,

(Ol) $X(\cdot, t)$ satisfies the rephrased Oleinik condition $(4.21)$ on $(0,1)$ and for all $t>0$

(Eq) $X(\cdot, t)$ satisfies (4.2) almost everywhere in $z \in[0,1]$ and for all $t>0$.

We now state the main result of our paper.

Theorem 5.2 (Existence and uniqueness of measures solutions). Let $f_{I} \in \mathcal{P}(\mathbb{R}) \cap$ $B V(\mathbb{R})$ have connected compact support. Then there exists a unique global-intime entropy measure $[0,+\infty) \ni t \mapsto \mu(t) \in \mathcal{M}_{0}$ solution to (2.2) with initial datum $f_{I}$ in the sense of Definition 5.1. Moreover, the absolutely continuous part $\rho \in L^{\infty}\left([0, \infty) ; L^{1}(\mathbb{R})\right)$ of $\mu(t)$ is given by the change of variables (3.7) where $u$ is the unique entropy solution to (3.8)-(3.9) in the sense of Definition 3.2.

Proof. Step 1: CONSTRUCTION OF $u$. Let $\operatorname{supp}\left(f_{I}\right)=(A, B)$. For simplicity we shall assume $A<0<B$, but the proof can be easily repeated in the case of $a$ and $b$ having the same sign. Let

$$
u_{I}(\xi):=(\gamma|\xi|)^{1 / \gamma} f_{I}\left(\frac{\operatorname{sign}(\xi)}{1+\gamma}(\gamma|\xi|)^{(1+\gamma) / \gamma}\right)
$$

Notice that $m_{L}:=\int_{-\infty}^{0} u_{I}(\xi) d \xi=\int_{-\infty}^{0} f_{I}(x) d x$. Moreover, as $f_{I} \in L^{\infty}$, then $u_{I}$ is continuous at $\xi=0$ with $u_{I}(0)=0$. Let $u_{L}$ and $u_{R}$ be the unique (global in time) entropy solutions to (3.8) and (3.9) provided by Theorem 3.3 with initial conditions $u_{I, L}=\left.u_{I}\right|_{\xi<0}$ and $u_{I, R}=\left.u_{I}\right|_{\xi>0}$ respectively. Let $u$ be defined as in (3.10). By a simple comparison argument, in view of Remark 3.4, we infer that $u(\xi)>0$ if and only if $\xi \in I(t)=(a(t), 0) \cup(0, b(t))$ for some continuous functions $a(t)$ and $b(t)$. We set

$$
t^{*}=\sup \{t \geq 0: u(\cdot, t) \text { is continuous at } \xi=0 \text { and } u(0, t)=0\} .
$$

Once again by a simple comparison argument, it is easily seen that $t^{*}>0$, since $u_{I}$ is continuous at $\xi=0$, and therefore the $u=0$ datum at $\xi=0$ is 'transported' for at least some short time, until possible shocks may occur at $\xi=0$. Let

$$
M_{L}(t):=\int_{-\infty}^{0} u_{L}(\xi, t) d \xi, \quad M_{R}(t):=\int_{0}^{+\infty} u_{R}(\xi, t) d \xi .
$$

We remark that $M_{L}(t)$ and $M_{R}(t)$ are constant with $M_{L}(t)+M_{R}(t)=1$ on $0 \leq$ $t \leq t^{*}$.

Step 2: CONSTRUCTION OF $\rho$. Now, let us set

$$
\begin{aligned}
& \rho_{L}(x, t)=\xi^{\prime}(x) u_{L}(\xi(x), t) \quad \text { on } x<0 \\
& \rho_{R}(x, t)=\xi^{\prime}(x) u_{R}(\xi(x), t) \quad \text { on } x>0
\end{aligned}
$$


with $\xi(x)$ defined in (3.6). Notice that $\operatorname{supp}[\rho(\cdot, t)] \backslash\{0\}=(A(t), B(t)) \backslash\{0\}$ for all times, with

$$
\begin{aligned}
& A(t)=-\frac{1}{1+\gamma}(\gamma|a(t)|)^{\frac{1+\gamma}{\gamma}} \\
& B(t)=\frac{1}{1+\gamma}(\gamma|b(t)|)^{\frac{1+\gamma}{\gamma}} .
\end{aligned}
$$

By comparison with the solution $\tilde{u}$ to (3.8)-(3.9) with initial condition

$$
\tilde{u}_{I}=\left.(\gamma|\xi|)^{1 / \gamma} \sup \left(f_{I}\right) \chi\right|_{(a(0), b(0))},
$$

from (5.4) we easily get

$$
\rho(x, t) \leq C|x|^{-1 /(1+\gamma)}\left(|x|^{\gamma /(1+\gamma)}\right)^{1 / \gamma}=C
$$

for $t$ small enough, which means that $\rho(\cdot, t) \in L^{\infty}$ for small times (see Example 3.6 for more details). On the other hand, since $\xi^{\prime}(x)$ blows up at $x=0, \rho$ may develop a blow-up at $x=0$ at time $t=t^{*}$ (see Theorem 6.1 below). However, we can estimate

$$
\rho(x, t) \leq\left|\xi^{\prime}(x)\right|\|u(\cdot, t)\|_{L^{\infty}(\mathbb{R})} \leq\left|\xi^{\prime}(x)\right|\left\|u_{I}\right\|_{L^{\infty}(\mathbb{R})} \leq C|x|^{-1 / 1+\gamma},
$$

where we have used $u_{I} \in L^{\infty}$, which is a trivial consequence of the definition of $u_{I}$ and of $f_{I}$ having compact support. As $\rho(\cdot, t)$ is compactly supported for all times, then (5.5) shows that $\rho(\cdot, t) \in L^{1}(\mathbb{R})$ for all $t \geq 0$. Moreover, $x \rho^{1+\gamma} \in L^{\infty}(\mathbb{R})$. We claim that $\rho$ is a weak solution to (3.2) in the sense if Definition 3.1. To see this, we have to verify that $\rho$ satisfies (3.1) for all $\phi \in C_{c}^{\infty}(\mathbb{R} \times[0,+\infty))$ with $\phi(0, t)=0$. The first and the second term in (3.1) scale in a straightforward way. By using (3.6), we now compute, for $\widetilde{\phi}(\xi, t)=\phi(x(\xi), t)$,

$$
\begin{aligned}
& \int_{0}^{+\infty} \int_{0}^{+\infty} \rho^{1+\gamma}(x, t) x \phi_{x}(x, t) d x d t \\
& =\int_{0}^{+\infty} \int_{0}^{+\infty} \xi^{\prime}(x)^{1+\gamma} x u_{R}^{1+\gamma}(\xi, t) \phi_{x}(x, t) d x d t \\
& =\int_{0}^{+\infty} \int_{0}^{+\infty}[(1+\gamma) x]^{-1} x u_{R}^{1+\gamma}(\xi(x), t) \xi^{\prime}(x) \widetilde{\phi}_{\xi}(\xi(x), t) d x d t \\
& =\int_{0}^{+\infty} \int_{0}^{+\infty}(1+\gamma)^{-1} u_{R}^{1+\gamma}(\xi, t) \widetilde{\phi}_{\xi}(\xi, t) d \xi d t,
\end{aligned}
$$

and this term, combined with the other two terms and with the definition of weak solution for $u$, and with the terms obtained on $x<0$, gives the desired formula (3.1). Since $u$ is $B V(\mathbb{R})(c f$. [8]), the right and left limits of $u$ are always defined, and $u$ has at most a countable number of jumps. The jumps are decreasing on $\xi<0$ 
and increasing on $\xi>0$ since $u$ is an entropy solution. The scaling $u \rightarrow \rho$ clearly preserves the ordering of the jumps, and hence all the jumps of $\rho$ are admissible. We stress that the $\rho$ introduced here is globally defined for all times. Such $\rho$ represents the absolutely continuous part of the candidate measure solution to our problem.

STEP 3: CONSTRUCTION OF THE CUMULATIVE Distributions. As $\rho \in L^{1}(\mathbb{R})$, we can define the cumulative distributions $F_{L}$ and $F_{R}$ as in (4.18) and (4.17). Please notice that $F_{L}$ (respectively $F_{R}$ ) is a strictly increasing bijection from $[A(t), 0)$ (respectively $(0, B(t)])$ onto $\left[0, M_{L}(t)\right)$ (respectively $\left.\left(0, M_{R}(t)\right]\right)$. Moreover, $F_{R}$ and $F_{L}$ are defined globally in time. Let $0<t_{1}<t_{2}$ and let us apply Definition 3.1 with the test functions $\varphi_{1}^{\epsilon}(x, t)=\chi_{\left[t_{1}, t_{2}\right]}^{\epsilon}(t) \chi_{\left(-\infty, x_{0}\right]}^{\epsilon}(x)$ for $x_{0}<0$ and $\varphi_{2}^{\epsilon}(x, t)=\chi_{\left[t_{1}, t_{2}\right]}^{\epsilon}(t) \chi_{\left[x_{0},+\infty\right)}^{\epsilon}(x)$ for $x_{0}>0$, where $\chi^{\epsilon}$ is a standard mollification of a characteristic function. After sending $\epsilon \rightarrow 0$, multiplying by $\left(t_{2}-t_{1}\right)^{-1}$, and sending $t_{2} \searrow t_{1}$ we get

$$
\begin{aligned}
& \frac{\partial F_{L}\left(x_{0}, t_{1}\right)}{\partial t}=x_{0} \rho_{L}\left(x_{0}, t_{1}\right)^{1+\gamma} \quad x_{0}<0 \\
& \frac{\partial F_{R}\left(x_{0}, t_{1}\right)}{\partial t}-M_{R}^{\prime}(t)=x_{0} \rho_{R}\left(x_{0}, t_{1}\right)^{1+\gamma} \quad x_{0}>0 .
\end{aligned}
$$

STEP 4: DEFINITION OF THE CANDIDATE SOLUTION $X(z, t)$. Let us set

$$
\begin{aligned}
& X_{L}(z, t)=\inf \left\{x: F_{L}(x, t)>z\right\} \quad \text { on } 0 \leq z<M_{L}(t) \\
& X_{R}(z, t)=\inf \left\{x: 1-M_{R}(t)+F_{R}(x, t)>z\right\} \quad \text { on } 1-M_{R}(t)<z<1 \\
& X(z, t)= \begin{cases}X_{L}(z, t) & \text { on } 0 \leq z<M_{L}(t) \\
0 & \text { on } M_{L}(t) \leq z \leq 1-M_{R}(t) \\
X_{R}(z, t) & \text { on } 1-M_{R}(t)<z<1 .\end{cases}
\end{aligned}
$$

The candidate solution $\mu(t) \in \mathcal{M}_{0}$ is defined as $\mu(t)=\partial_{x} F(\cdot, t)$ in the sense of distributions, with $F(x, t)=\inf \{z \in[0,1]: X(z, t)>x\}$ being the pseudo inverse of $X$. Notice in particular that $X=X_{\mu}$ according to (5.1). It is clear from the above definition that $\mu(t) \in \mathcal{M}_{0}$, with $\mu(t)=m(t) \delta_{0}+\rho(\cdot, t) \mathcal{L}_{1}$, which proves that the absolutely continuous part of $\mu(t)$ is $\rho(t)$ given in Step 3 .

As $f_{I} \in \mathcal{M}_{0}$, then $X(\cdot, 0) \in \mathcal{X}_{0}$, and the property (In) is trivially proven. Now, as $X_{L}(\cdot, t)$ and $X_{R}(\cdot, t)$ are both strictly increasing, we have that $X_{L}(\cdot, t)$ : $\left[0, M_{L}(t)\right) \rightarrow[a(t), 0)$ is the inverse of $F_{L}(\cdot, t)$ and $X_{R}(\cdot, t):\left(1-M_{R}(t), 1\right] \rightarrow$ $(0, b(t)]$ is the inverse of $1-M_{R}(t)+F_{R}(\cdot, t)$. Therefore,

$$
\begin{aligned}
& \partial_{z} X_{L}(z, t)=\left(\rho_{L}\left(X_{L}(z, t), t\right)\right)^{-1} \quad z \in\left(0, M_{L}(t)\right) \\
& \partial_{z} X_{R}(z, t)=\left(\rho_{R}\left(X_{R}(z, t), t\right)\right)^{-1} \quad z \in\left(1-M_{R}(t), 1\right) .
\end{aligned}
$$

Moreover

$$
\begin{array}{ll}
\partial_{t} X_{L}(z, t)=-\partial_{z} X_{L}(z, t) \partial_{t} F_{L}(X(z, t) t) & z \in\left(0, M_{L}(t)\right) \\
\partial_{t} X_{R}(z, t)=-\partial_{z} X_{L}(z, t) \partial_{t}\left(F_{R}(X(z, t), t)-M_{R}(t)\right) & z \in\left(1-M_{R}(t), 1\right) .
\end{array}
$$


We can then combine (5.6)-(5.7) with (5.9)-(5.12) to obtain that (4.2) is satisfied on the set $z \in\left(0, M_{L}(t)\right) \cup\left(1-M_{R}(t), 1\right)$. Since $X$ defined in (5.8) is constantly zero in the remaining set $z \in\left[M_{L}(t), 1-M_{R}(t)\right]$, then the property (Eq) is satisfied.

STEP 5: REgUlarity. As for the property (Re), it is clear that $X(\cdot, t)$ is continuous on $z \notin\left[M_{L}(t), 1-M_{R}(t)\right]$. Moreover, since $F_{L}(0, t)=M_{L}(t)$ and $F_{R}(0, t)=0$, then $X_{L}\left(M_{L}(t)^{-}, t\right)=0=X_{R}\left(\left(1-M_{R}(t)\right)^{+}, t\right)$, which implies continuity on the whole set $[0,1]$. Moreover, $X(\cdot, t)$ is bounded, and nonincreasing. Since $u(\cdot, t) \in B V(\mathbb{R})$ for all times, then $\rho(\cdot, t) \in B V_{\text {loc }}(\mathbb{R} \backslash\{0\})$, and therefore the property (X3) is proven. Since $u$ is an entropy solution in the sense of Definition 3.2, an increasing jump at $\xi=a(t)$ is not entropic, and therefore the same holds for $\rho(\cdot, t)$ at $x=A(t)$. Therefore, $\rho(\cdot, t)$ is continuous at $x=A(t)$ with $\rho(A(t), t)=0$. Similarly, one can prove that $\rho(B(t), t)=0$. This proves properties (X4) and (X5). Thus, $(\mathrm{Re})$ is proven.

Finally, we prove the property $(\mathrm{Ol})$, namely that the Oleinik condition (4.21) is satisfied. Let $z_{0}$ a point of jump for $X_{z}(\cdot, t)$, and let $x_{0}=X\left(z_{0}, t\right)$. Assume first that $x_{0}<0$. It is clear that $\rho_{L}$ has a jump at $x_{0}$. Since $\rho$ is entropic, the jump is decreasing. Since $X_{z}\left(z_{0}^{-}, t\right)=\left(\rho_{L}\left(x_{0}^{-}, t\right)^{-1}\right.$ has an increasing jump, as required in (4.21). A similar computation holds in the case $x_{0}>0$.

STEP 6: UNIQUENESS. Finally, we prove uniqueness. Let $\widetilde{X}$ be another solution in the sense of Definition 5.1 with initial condition $f_{I}$. Let

$$
\begin{array}{rlrl}
I_{L}(t) & =\{z \in[0,1]: \widetilde{X}(z, t)<0\} & \\
I_{R}(t) & =\{z \in[0,1]: \widetilde{X}(z, t)>0\} & \\
\widetilde{F}_{L}(x, t) & =\inf \left\{z \in I_{L}(t): \widetilde{X}(z, t)>x\right\} & & \text { on } x<0 \\
\widetilde{F}_{R}(x, t) & =\inf \left\{z \in I_{R}(t): \widetilde{X}(z, t)>x\right\} & & \text { on } x>0 .
\end{array}
$$

Both $\widetilde{F}_{L}$ and $\widetilde{F}_{R}$ are strictly increasing on their respective domains because of property (X1). As a consequence of (4.2), a simple computation shows that

$$
\partial_{t} \widetilde{F}_{L}(x, t)=\left(\partial_{x} \widetilde{F}_{L}\right)^{1+\gamma} x,
$$

for all $x<0$. Therefore, $\widetilde{\rho}_{L}:=\widetilde{F}_{L, x}$ satisfies (3.2) on $x<0$, and a symmetric argument shows that $\widetilde{\rho}_{R}:=\widetilde{F}_{R, x}$ satisfies (3.2) on $x>0$. Let

$$
\widetilde{\rho}(x, t)= \begin{cases}\widetilde{\rho}_{L}(x, t) & \text { if } x<0 \\ \widetilde{\rho}_{R}(x, t) & \text { if } x>0\end{cases}
$$

and let $\widetilde{u}$ be defined by $\widetilde{u}(\xi, t)=x(\xi) \widetilde{\rho}(x(\xi), t)$ with $x(\xi)$ given as in (3.3). The usual scaling computation gives that $\widetilde{u}$ is a weak entropy solution to (3.8)-(3.9) in the sense of Definition 3.2. The fact that $\tilde{u}$ satisfies the entropy condition (3.11) comes from property (4.21) and from the reversed argument at the end of Step 5 above. Therefore, as $\widetilde{X}(z, 0)=X(z, 0)$ implies that $u$ and $\widetilde{u}$ have the same 
initial condition, the result in Theorem 3.3 implies that $u \equiv \tilde{u}$. This also implies that $I_{L}(t)=\left[0, M_{L}(t)\right)$ and $I_{R}(t)=\left(1-M_{R}(t), 1\right]$, which imply also that $X$ and $\widetilde{X}$ coincide on the set $z \neq 0$. Now, at each time $t$ there are two possible situations: either $I_{L}(t) \cup I_{R}(t)=[0,1]$, or $I_{L}(t) \cup I_{R}(t) \varsubsetneqq[0,1]$. In the former case, $\widetilde{X}(\cdot, t) \equiv X(\cdot, t)$ on $[0,1]$. In the latter case, as we clearly have $\tilde{X}(z, t) \nearrow 0$ as $z \nearrow \sup I_{L}(t)$ and $\widetilde{X}(z, t) \searrow 0$ as $z \searrow \inf I_{R}(t)$, the only possible way to extend $\widetilde{X}$ on the set $[0,1] \backslash\left(I_{L}(t) \cup I_{R}(t)\right)$ in a way such that $\widetilde{X}(\cdot, t)$ is non-decreasing is to set $\widetilde{X}(z, t)=0$ on $z \in[0,1] \backslash\left(I_{L}(t) \cup I_{R}(t)\right)$. Therefore, $\widetilde{X}$ coincides with $X$ defined in (5.8), and the proof is complete.

Corollary 5.3 (Result in terms of the original equation). Let $f_{I} \in \mathcal{P}(\mathbb{R}) \cap B V(\mathbb{R})$ have connected compact support. Then there exists a unique curve $[0,+\infty) \ni t \mapsto$ $v(t) \in \mathcal{M}_{0}$ such that the corresponding pseudo inverse variable $X(z, t)$ satisfies $X(\cdot, t) \in \mathcal{X}_{0}^{+}$for all times $t \geq 0$, it is a solution to the pseudo inverse version of $(1.1)$

$$
\partial_{t} X=-X\left(1+\frac{1}{X_{z}^{\gamma}}\right)
$$

such that $\left.v\right|_{t=0}=f_{I} \mathcal{L}_{1}$, and such that $X$ satisfies the rephrased Oleinik condition (4.21) on $(0,1)$ and for all $t>0$.

Remark 5.4. In Theorem 5.2 we have assumed for simplicity that the support of the initial condition has just one connected component. Such an assumption could be easily removed by considering a finite number of components, and then generalizing the above result to all $B V$ initial probability densities with compact support by approximation. We omit the details.

\section{Qualitative and asymptotic behaviour}

We now prove that all measure solutions to (3.2) develop a singularity in finite time, and that the concentrated mass is strictly increasing in time, and it converges to the total mass for large times.

Theorem 6.1 (Finite time blow up). Let $f_{I} \in \mathcal{P}(\mathbb{R}) \cap B V(\mathbb{R})$ with connected compact support. Then, the unique global-in-time entropy measure solution $\mu(t)=$ $m(t) \delta_{0}+\rho(\cdot, t) \mathcal{L}_{1}$ to $(2.2)$ with initial datum $f_{I}$ in the sense of Definition 5.1 satisfies the following properties:

(FTBU) There exists a time $T<+\infty$ such that $\rho(\cdot, t) \notin L^{\infty}(\mathbb{R})$ for all $t \geq T$;

(SCon) The function $m(\cdot)$ is continuous and strictly increasing in time, and it satisfies $\lim _{t \rightarrow+\infty} m(t)=1$.

Proof. The proof relies on an extensive use of the comparison principle 3.4. Let $\operatorname{supp}\left(f_{I}\right)=(a, b)$, and assume for simplicity that $b>0$. Let $[c, d] \subset(0, b)$ such 
that $\lambda:=\min _{x \in[c, d]} f_{I}(x)>0$. We define $u_{I}(\xi)$ as in the proof of Theorem 5.2,

$$
u_{I}(\xi):=(\gamma|\xi|)^{1 / \gamma} f_{I}\left(\frac{\operatorname{sign}(\xi)}{1+\gamma}(\gamma|\xi|)^{(1+\gamma) / \gamma}\right)
$$

and consider $u$ the unique entropy solution to (3.8)-(3.9) with initial condition $u_{I}$. Let

$$
\bar{u}_{0}(\xi):=\lambda(\gamma|\xi|)^{1 / \gamma} \chi_{[c, d]},
$$

and consider the corresponding entropy solution $\bar{u}_{R}$ to (3.8) on $\xi>0$. We next show that $\bar{u}\left(0^{+}, t\right)$ becomes nonzero in a finite time $t^{*}$. The solution $\bar{u}$ has an increasing shock wave $\xi=s(t)$ originating at $\xi=c$, with velocity $-\bar{u}(s(t))^{\gamma} /(1+$ $\gamma)$. At $\xi=d$, a rarefaction wave is originated with left front given by the straight line $\xi(t)=d-t \lambda^{\gamma} \gamma d$, i.e. the rarefaction wave is faster than the shock wave. If the shock wave reaches $\xi=0$ before the rarefaction wave does, then the assertion is proven. Otherwise, another shock wave is originated, which can be easily computed to travel with a law of order $-C t^{1 / \gamma}$ for large times. Hence, the rarefaction wave reaches zero in a finite time, and the assertion that $\bar{u}\left(0^{+}, t\right)$ becomes nonzero in a finite time $t^{*}$ is proven. Now, we employ the comparison principle in Remark 3.4 to deduce that $u\left(0^{+}, t\right)$ also becomes nonzero at least for $t \geq t^{*}$. Now, if $\mu(t)$ has already developed a blow-up singularity in $L^{\infty}$ before $t=t^{*}$, then (FTBU) is trivially true. Assume this is not the case, then we know from the proof of Theorem 5.2 that $\rho$ defined in (5.4) is the unique entropy solution to (3.2), and the above computations show that $\lim _{x \searrow 0^{+}} \rho\left(x, t^{*}\right)=+\infty$, which proves (FTBU).

Now, a trivial application of the definition of weak solution for $u$ (with suitable choice of the test functions and with suitable mollifiers) gives, for all $s>t$

$$
\int_{0}^{+\infty} u(\xi, s) d \xi-\int_{0}^{+\infty} u(\xi, t) d \xi=-\int_{t}^{s} u^{1+\gamma}\left(0^{+}, \sigma\right) d \sigma .
$$

Now, let $t_{1}=\sup \{t>0: \quad m(t)=0\}$. The support of $u(\cdot, t)$ is a connected interval, and we know that $u\left(0^{+}, t_{1}\right)>0$. To see this, assume by contradiction that this is not the case, then there exists an interval $(0, \delta)$ on which $u(\cdot, t)$ is zero, and by a simple characteristics argument it is easy to see that $u\left(0^{+}, t_{1}\right)$ will remain zero for some short time $\left[t_{1}, t_{1}+\epsilon\right)$ with $\epsilon>0$, which contradicts the maximality of $t_{1}$. Therefore, $\inf \operatorname{supp}\left(u_{R}(\cdot, t)\right)=0$ for all $t \geq t_{1}$. Once again, a very simple characteristics argument shows that $u\left(0^{+}, t\right)>0$ for all $t \geq t_{1}$, as in that point $u$ will achieve values originated from the interior of $\operatorname{supp}\left(u\left(\cdot, t_{1}\right)\right)$. Hence, (6.1) implies that

$$
\int_{0}^{a(s)} u(\xi, s) d \xi<\int_{0}^{a(t)} u(\xi, t) d \xi
$$

which gives

$$
\int_{0}^{+\infty} \rho(x, s) d x<\int_{0}^{+\infty} \rho(x, t) d x
$$


and hence $m(t)$ is increasing by conservation of the total mass. To finish the proof of (SCon), we need to prove that all the mass concentrates as $t \rightarrow+\infty$. To prove this assertion, let $C=\left\|f_{I}\right\|_{L^{\infty}}$, and let $[A, B] \supset \operatorname{supp}\left(f_{I}\right)$. Define $\bar{f}(x)=C \chi_{[A, B]}$, and consider

$$
\tilde{u}_{I}(\xi):=(\gamma|\xi|)^{1 / \gamma} \bar{f}\left(\frac{\operatorname{sign}(\xi)}{1+\gamma}(\gamma|\xi|)^{(1+\gamma) / \gamma}\right) .
$$

Let $\widetilde{u}(\xi, t)$ be the entropy solution in the sense of Definition 3.2 with initial condition $\tilde{u}$. A simple characteristic argument shows that $\lim _{t \rightarrow+\infty}\|\tilde{u}(\cdot, t)\|_{L^{\infty}}=0$. Hence, by the usual comparison argument, $\lim _{t \rightarrow+\infty}\|u(\cdot, t)\|_{L^{\infty}}=0$. Still by a comparison argument, one easily sees that $\operatorname{supp}(u(\cdot, t))$ is uniformly bounded in time, and this implies

$$
\int_{\mathbb{R}} u(\xi, t) d \xi \rightarrow 0, \quad \text { as } t \rightarrow+\infty,
$$

From the proof of Theorem 5.2, $\rho$ defined in (5.4) from $u$ is the absolutely continuous part of $\mu(t)$, and we have

$$
\int_{\mathbb{R}} \rho(x, t) d x=\int_{\mathbb{R}} u(\xi, t) d \xi \rightarrow 0, \quad \text { as } \quad t \rightarrow+\infty,
$$

which implies the assertion.

As a straightforward consequence of the above proof, we have the following:

Corollary 6.2. Under the same assumptions of Theorem 6.1, the support of $\mu(t)$ is uniformly bounded in time.

Remark 6.3. The strict monotonicity of $m(t)$ would be clearly violated in case of solutions with many components in their support. In that case, the concentrated mass would stay constant for some waiting time until a new wave will hit the boundary $x=0$.

Remark 6.4 (Asymptotic behaviour for the original equation (1.1)). A similar statement to that in Theorem 6.1 can be formulated for the global measure solution to (1.1) obtained in Corollary 5.3. Once again, the same qualitative properties can be easily recovered via the scaling (2.1). Additionally, the measure of the support of all measure solutions of (1.1) converges algebraically to zero for large times. As a consequence of the scaling (2.1) and of Corollary 6.2, we can easily see that

$$
W_{p}\left(\delta_{0}, f(t)\right) \leq C(1+t)^{-1 / \gamma},
$$

for some $C>0$, and for all $1 \leq p \leq \infty$. Here $W_{p}$ denotes the $p$-Wasserstein distance, see e.g. [29]. $f(t)$ is, by abuse of notation, the global-in-time measure solution to (1.1) obtained in Corollary 5.3. 


\section{References}

[1] C. BARdos, A. Y. LE RouX and J.-C. NÉdÉLEC, First order quasilinear equations with boundary conditions, Comm. Partial Differential Equations 4 (1979), 1017-1034.

[2] N. Ben Abdallah, I. M. Gamba and G. Toscani, On the minimization problem of sub-linear convex functionals, Kinet. Relat. Models 4 (2011), 857-871.

[3] J. A. CARrillo, P. LAurenÇOT and J. Rosado, Fermi-Dirac-Fokker-Planck equation: well-posedness \& long-time asymptotics, J. Differential Equations 247 (2009), 2209-2234.

[4] J. A. CARrillo, J. Rosado and F. SAlvarani, ID nonlinear Fokker-Planck equations for fermions and bosons, Appl. Math. Lett. 21 (2008), 148-154.

[5] J. A. CARRILlO and G. TOSCANI, "Wasserstein Metric and Large-time Asymptotics of Nonlinear Diffusion Equations", New trends in mathematical physics, World Sci. Publ., Hackensack, NJ, 2004, 234-244.

[6] S. Chapman and T. G. Cowling, "The Mathematical Theory of Nonuniform Gases", Cambridge Mathematical Library, Cambridge University Press, Cambridge, third edition, 1990.

[7] M. G. Crandall and P.-L. LiOns, Viscosity solutions of Hamilton-Jacobi equations, Trans. Amer. Math. Soc. 277 (1983), 1-42.

[8] C. M. Dafermos, "Hyperbolic Conservation Laws in Continuum Physics", Grundlehren der Mathematischen Wissenschaften, Vol. 325, Springer-Verlag, Berlin, third edition, 2010.

[9] F. DUBOIS and P. LEFLOCH, Boundary conditions for nonlinear hyperbolic systems of conservation laws, In: "Nonlinear Hyperbolic Equations - Theory, Computation Methods, and Applications" (Aachen, 1988), Notes Numer. Fluid Mech., Vol. 24, Vieweg, Braunschweig, 1989, 96-104.

[10] M. Escobedo, M. A. Herrero and J. J. L. Velazquez, A nonlinear Fokker-Planck equation modelling the approach to thermal equilibrium in a homogeneous plasma, Trans. Amer. Math. Soc. 350 (1998), 3837-3901.

[11] M. EsCOBEDO and S. MisCHLER, Équation de Boltzmann quantique homogène: existence et comportement asymptotique, C. R. Acad. Sci. Paris Sér. I Math. 329 (1999), 593-598.

[12] M. ESCOBEDO and S. MisCHLER, On a quantum Boltzmann equation for a gas of photons, J. Math. Pures Appl. (9) 80 (2001), 471-515.

[13] M. Escobedo, S. Mischler and M. A. Valle, "Homogeneous Boltzmann Equation in Quantum Relativistic Kinetic Theory”, Electronic Journal of Differential Equations, Monograph, Vol. 4, Southwest Texas State University, San Marcos, TX, 2003.

[14] M. Escobedo and J. J. L. VelázQuez, On the blow up of supercritical solution of the Nordheim equation for bosons, Comm. Math, Phys. 330 (2014), 331-365.

[15] D. HofF, The sharp form of Oleinik's entropy condition in several space variables, Trans. Amer. Math. Soc. 276 (1983), 707-714.

[16] C. Josserand, Y. Pomeau and S. Rica, Self-similar singularities in the kinetics of condensation, J. Low Temperature Phys. 145 (2006), 231-265.

[17] H. LI and G. TosCANI, Long-time asymptotics of kinetic models of granular flows, Arch. Ration. Mech. Anal. 172 (2004), 407-428.

[18] G. KANIADAKIS and P. QUARATI, Ckinetic equation for classical particles obeying an exclusion principle, Phys. Rev. E 48 (1993), 4263-4270.

[19] G. Kaniadakis and P. QUARATI, Classical model of bosons and fermions, Phys. Rev. E 49 (1994), 5103-5110.

[20] A. S. KompaneETS, The establishment of thermal equilibrium between quanta and electrons, Soviet Phys. JETP 4 (1957), 730-737.

[21] S. N. KRUŽKOV, First order quasilinear equations with several independent variables, Mat. Sb. (N.S.) 81 (1970), 228-255.

[22] R. LaCAZE, P. Lallemand, Y. Pomeau and S. RicA, Dynamical formation of a BoseEinstein condensate, Phys. D 152/153 (2001), 779-786. 
[23] P.-L. Lions, "Generalized Solutions of Hamilton-Jacobi Equations", Research Notes in Mathematics, Vol. 69, Pitman (Advanced Publishing Program), Boston, Ma., 1982.

[24] O. A. OlEINIK, Discontinuous solutions of nonlinear differential equations, Amer. Math. Soc. Transl. (2) 26 (1963), 95-172.

[25] D. V. Semikov and J. I. TKacheV, Kinetics of bose condensation, Phys. Rev. Lett. 74 (1995), 3093-3097.

[26] D. V. Semikov and J. I. TKachev, Condensation of bosons in the kinetic regime, Phys. Rev. D 55 (1997), 489-502.

[27] H. Spohn, Kinetics of the Bose-Einstein condensation, Phys. D 239 (2010), 627-634.

[28] G. TosCANI, Finite time blow up in Kaniadakis-Quarati model of Bose-Einstein particles, Comm. Partial Differential Equations 37 (2012), 77-87.

[29] C. VILlani, "Topics in Optimal Transportation", Graduate Studies in Mathematics, Vol. 58, American Mathematical Society, Providence, RI, 2003.

\author{
Department of Mathematics \\ Imperial College London \\ South Kensington Campus \\ London SW7 2AZ, Great Britain \\ carrillo@imperial.ac.uk \\ Mathematical Sciences \\ University of Bath \\ Claverton Down Bath \\ BA2 7AY, Great Britain \\ m.difrancesco@bath.ac.uk \\ Dipartimento di Matematica \\ Università di Pavia \\ Via Ferrata, 1 \\ 27100 Pavia, Italia \\ giuseppe.toscani@unipv.it
}

